\title{
Idade e ingestão dietética de cálcio por mulheres sobreviventes de câncer de mama: um fator adicional de risco para o desenvolvimento da osteoporose?
}

Age and calcium intake in breast cancer survivors: an additional risk factor for osteoporosis?

\author{
Soraia Pinheiro Machado', Maria Fernanda Montenegro Cavalcante², Maria Olganê Dantas Sabry², Luís Gonzaga Porto Pinheiro³, \\ Helena Alves de Carvalho Sampaio ${ }^{4}$
}

\section{Resumo}

Pacientes sobreviventes de câncer de mama apresentam risco aumentado para osteoporose, como efeito das drogas antineoplásicas e pela não utilização de reposição hormonal. Este estudo pretendeu investigar se a idade e o consumo dietético de cálcio aparecem como fatores de risco adicionais ao desenvolvimento da osteoporose num grupo de mulheres sobreviventes de câncer de mama. Foram investigadas 59 mulheres, em serviços públicos de mastologia de Fortaleza. Foram considerados fatores de risco a idade de 45 anos ou mais e a ingestão de cálcio inferior a 100\% das necessidades diárias (DRI). A idade (média de 49,14 anos) e o baixo consumo de cálcio, detectado em $86,44 \%$ do grupo, constituíram-se fortes fatores contribuintes para o risco de desenvolvimento da osteoporose. Conclui-se que o grupo necessita de ações educativas para o aumento do consumo de fontes alimentares de cálcio, como estratégia para reduzir um dos fatores de risco para osteoporose presente nesse tipo de paciente. Palavras-chave: Osteoporose; Câncer de mama; Consumo de cálcio

1Mestrado Acadêmico em Saúde Pública - Universidade Estadual do Ceará

2Departamento de Nutrição da Universidade Estadual do Ceará

3Faculdade de Medicina da Universidade Federal do Ceará

4Universidade Estadual do Ceará

Endereço para correspondência: Rua J da Penha, 332/1002, Centro, Fortaleza - Ceará. CEP: 60110-120. E-mail: soraiamachado@yahoo.com.br 


\section{INTRODUÇÃO}

A incidência da osteoporose e das fraturas associadas tem crescido dramaticamente nas últimas décadas, tornando-se um dos maiores problemas de saúde pública no mundo, especialmente nos países ocidentais. Esse crescimento tende a continuar de maneira acelerada, devido ao progressivo aumento da população idosa ${ }^{1}$. A osteoporose é um distúrbio metabólico, caracterizada pela perda progressiva e acelerada da massa óssea e deterioração da microarquitetura do tecido ósseo, induzindo sua fragilidade e aumentando o risco de fraturas ${ }^{2}$. Após atingir o pico de massa óssea, os indivíduos iniciam uma perda, que varia de $0,3 \%$ a $0,5 \%$ a cada ano $^{3}$. Dessa forma, a idade aparece como o maior contribuinte do risco de fraturas ${ }^{3-10}$. Entre os indivíduos afetados de idade mais elevada, destacam-se as mulheres na pós-menopausa, devido ao hipoestrogenismo decorrente da falência ovariana ${ }^{3,10-14}$. Além desse fator, outros fatores genéticos e ambientais exercem importante papel na saúde óssea. Dentre os ambientais, destacamse: nutrição, exercício físico, estilo de vida, doenças e medicamentos ${ }^{8,10}$.

Considerando o sexo feminino, surge um outro fator que pode aumentar ainda mais o risco de osteoporose, mesmo que ainda não estejam menopausadas. É o caso de mulheres com câncer de mama tratadas com agentes quimioterápicos. Tais agentes, se aplicados nas mulheres na pré-menopausa, levam à redução da função ovariana, acarretando uma menopausa precoce. Verifica-se, portanto, entre as sobreviventes dessa neoplasia, um risco aumentado de desenvolverem osteopenia ou osteoporose $\mathrm{e}^{11,14-20}$. Soma-se ainda o fato de que as mesmas são aconselhadas a não utilizarem ou suspenderem a terapia de reposição hormonal (TRH), uma das principais medidas de prevenção e tratamento da osteoporose para as mulheres, devido à potencial influência do estrogênio no crescimento do tumor ${ }^{14,18,21-23}$. Nessas mulheres, as complicaçoes decorrentes da perda óssea ocorrem mais cedo e com maior intensidade ${ }^{16,24}$.

Assim, torna-se importante acompanhar esse tipo de clientela quanto à sua massa óssea, bem como quanto à presença de outros fatores associados à osteoporose. Dentre tais fatores, merecem destaque os nutricionais, que agem desde o desenvolvimento do pico de massa óssea até a manutenção da massa óssea no adulto e a redução da perda óssea e de fraturas em idosos ${ }^{25}$.

Dentre os nutrientes mais estudados, como cálcio, vitamina $\mathrm{D}$, cafeína e outros, sem dúvida, destaca-se o cálcio $^{25-30}$.

É bem conhecido que uma ingestão adequada de cálcio é essencial para prevenir a osteoporose e suas fraturas decorrentes $3,4,7-10,18,25-27,30-40$, embora alguns autores não tenham comprovado tal fato em seus estudos $^{41-44}$. O suprimento adequado desse mineral desde a infância até o fim da vida é fundamental para a formação e a retenção de um esqueleto saudável ${ }^{39}$. Segundo Lau $\& \mathrm{Woo}^{26}$, a suplementação de cálcio produz um ganho de cerca de $4 \%$ de cálcio no esqueleto de adolescentes, quando administrada em doses de $500 \mathrm{mg} /$ dia, e reduz a perda óssea em mulheres na pós-menopausa em doses de $800 \mathrm{mg} /$ dia. Dados de Lunt et al. ${ }^{45}$ demonstram que um consumo adequado de leite em jovens protege contra deformidades vertebrais na velhice. O efeito benéfico da suplementação desse mineral sobre o osso é maior nas populações em que se verifica baixa ingestão do mesmo, e em mulheres que estão na menopausa há pelo menos cinco anos ${ }^{8,18}$. Heaney ${ }^{35}$ demonstrou que a suplementação de cálcio aumenta substancialmente a resposta do esqueleto à TRH em mulheres na pós-menopausa. Um estudo realizado na Suécia verificou um efeito positivo da ingestão de cálcio sobre a massa óssea apenas em níveis de ingestão superiores a $1600 \mathrm{mg} / \mathrm{dia}$; abaixo deste valor, não houve diferença na massa óssea dos indivíduos analisados ${ }^{46}$.

Diante do exposto, surgiu o interesse em investigar se o consumo de cálcio aparece como um fator de risco adicional ou de proteção no desenvolvimento da osteoporose num grupo de mulheres sobreviventes de câncer de mama. A identificação de mulheres com risco aumentado de desenvolverem osteoporose devido à insuficiente ingestão de cálcio permitirá a adoção de medidas preventivas, que contribuirão com o aumento da sobrevida e a melhora da qualidade de vida após a doença.

\section{METODOLOGIA}

O presente estudo integra uma pesquisa intitulada "Fatores constitucionais e ambientais associados ao câncer de mama na cidade de Fortaleza-Ceará", que vem sendo realizada sob a coordenação da Universidade Estadual do Ceará (UECE), com apoio da Fundação Cearense de Amparo à Pesquisa (FUNCAP), e que foi devidamente submetida e aprovada pelo Comitê de Ética em Pesquisa da UECE. Trata-se de um estudo transversal e descritivo, cuja coleta de dados ocorreu durante o ano de 2004. A população do estudo foi constituída de mulheres com diagnóstico prévio de câncer de mama, clientes dos Serviços de Mastologia do Instituto do Câncer do Ceará (ICC), da Maternidade Escola Assis Chateaubriand (MEAC) e do Instituto de Prevenção do Câncer do Ceará (IPCC). A amostra compreendeu 59 pacientes atendidas nas instituiçôes, residentes na Região Metropolitana da cidade de Fortaleza há pelo menos 
cinco anos e que concordaram em participar do estudo mediante a assinatura do Termo de Consentimento Livre e Esclarecido.

Todas responderam a um questionário semiestruturado, aplicado em seus respectivos locais de atendimento, contendo dados de identificação, socioeconômicos (nome, idade, escolaridade e renda familiar mensal) e sobre hábitos alimentares (recordatório alimentar de 24 horas e freqüência alimentar).

Os dados referentes ao consumo alimentar foram obtidos em medidas caseiras e transformados em gramas (sólidos) ou mililitros (líquidos), mediante padronização constante em Pinheiro et al. ${ }^{47}$ e quando ausente nestes, através do rótulo ou pesagem direta, esta realizada pelos pesquisadores, mediante aquisição dos alimentos em estabelecimentos comerciais locais, pesando-os em balança com capacidade para $500 \mathrm{~g}$ e sensibilidade de $10 \mathrm{~g}$. O conteúdo de cálcio dos alimentos foi determinado a partir da entrada dos dados alimentares em gramas ou mililitros no software Programa de Apoio à Decisão em Nutrição, versão 2,5a, da Escola Paulista de Medicina. A ingestão de cálcio assim obtida foi confrontada com as necessidades nutricionais diárias Dietary Reference Intake -DRI, que estabelecem um consumo diário de $1000 \mathrm{mg}$ para mulheres com idade entre 19 e 50 anos, e de $1200 \mathrm{mg}$ para mulheres com mais idade ${ }^{48}$. Calculou-se ainda o consumo de leite e derivados do grupo em número de porções da pirâmide alimentar brasileira, de acordo com Phillippi ${ }^{49}$. Os dados foram tabulados em Microsoft Excel 2002 para apresentação em frequiência simples e percentual, médias e variaçōes. Foi considerada presença de fator de risco para osteoporose a idade igual ou superior a 45 anos, de acordo com Kanis et al. ${ }^{50}$, e uma ingestão de cálcio menor do que a DRI.

\section{RESULTADOS}

O grupo estudado apresentou uma idade média de 49,14 anos (30 a 77 anos), estando sua distribuição em faixas etárias exibida na Tabela 1.

Tabela 1. Distribuição das mulheres estudadas segundo a faixa etária

\begin{tabular}{|c|c|c}
\hline Faixa etária (anos) & $\mathbf{n}$ & $\%$ \\
\hline$<35$ & 05 & 8,90 \\
$35-44$ & 20 & 33,90 \\
$45-54$ & 16 & 27,12 \\
$55-64$ & 11 & 18,64 \\
$\geq 65$ & 07 & 11,86 \\
\hline Total & 59 & 100,00 \\
\hline
\end{tabular}

O consumo dietético de cálcio foi deficiente $(<100 \%$ DRI) em $86,44 \%$ da amostra, tendo sido verificada uma média de consumo diário de 591,07mg (100,10mg $1719,56 \mathrm{mg}$ ). A Tabela 2 exibe as faixas de adequação do consumo de cálcio entre as mulheres entrevistadas.

Vale ressaltar ainda que, como pode ser observado no intervalo de variação de consumo de cálcio do grupo, nenhuma das mulheres estudadas ultrapassou o limite máximo considerado saudável $(2500 \mathrm{mg} / \mathrm{dia})$, o que poderia provocar hipercalciúria e hipercalcemia ${ }^{8}$.

Tabela 2. Distribuição das mulheres estudadas segundo a adequação do consumo de cálcio

\begin{tabular}{l|c|c}
\hline Adequação (\%) & $\mathbf{n}$ & $\%$ \\
\hline$<70$ & 43 & 72,88 \\
$70 \mid-100$ & 08 & 13,56 \\
$\geq 100$ & 08 & 13,56 \\
\hline Total & 59 & 100,00 \\
\hline
\end{tabular}

As fontes de cálcio mais citadas estão relacionadas na Tabela 3, observando-se pouca variação, com cada mulher consumindo apenas uma fonte alimentar rica no nutriente.

Tabela 3. Distribuição das mulheres estudadas segundo o consumo das principais fontes de cálcio

\begin{tabular}{l|c|c}
\hline \multicolumn{1}{c|}{ Fontes de cálcio } & $\mathbf{n}$ & $\%$ \\
\hline Leite integral & 24 & 40,70 \\
Leite em pó integral & 18 & 30,50 \\
Leite desnatado & 04 & 6,80 \\
Leite em pó desnatado & 03 & 5,10 \\
Queijo do sertáo & 08 & 13,60 \\
\hline
\end{tabular}

Quanto ao consumo de leite e derivados em porções da pirâmide alimentar (Tabela 4), verificou-se um elevado percentual de sobreviventes do câncer de mama relatando um consumo insuficiente ( $<3$ porçóes/dia), sendo a média de consumo diário do grupo de 1,59 porçōes ( 0,00 porção $-5,47$ porçôes).

Tabela 4. Distribuição das mulheres estudadas segundo o consumo de leite e derivados em porções recomendadas pela pirâmide alimentar brasileira

\begin{tabular}{c|c|c}
\hline Consumo (porções) & $\mathbf{n}$ & $\%$ \\
\hline$<2$ & 41 & 69,49 \\
$2 \mid-3$ & 10 & 16,95 \\
$\geq 3$ & 08 & 13,56 \\
\hline Total & 59 & 100,00 \\
\hline
\end{tabular}




\section{DISCUSSÃO}

Embora o grupo estudado tenha apresentado uma idade média inferior aos valores encontrados por estudos semelhantes realizados por Lindsey et al. ${ }^{18}$, de 54,03 anos, e por Tavani et al. ${ }^{51}$, de 55 anos, sugerindo ser o grupo do presente estudo mais jovem que a maioria dos grupos de pacientes e sobreviventes da neoplasia mamária, o mesmo exibe um fator de risco não modificável para osteoporose, pois a maioria $(57,62 \%)$ das mulheres tinha 45 anos ou mais. Estudos mostram que mais da metade das mulheres dessa faixa etária demonstram evidências da doença óssea nos exames de raio- $\mathrm{X}^{5}$ e que o risco de ocorrência de fraturas nas mesmas é oito vezes maior que o de mulheres mais joven $s^{50}$. Embora o presente estudo não tenha objetivado a detecção da presença de osteoporose, estes dados da literatura apontam que o grupo entrevistado é vulnerável a tais ocorrências.

O baixo consumo de produtos lácteos e, conseqüentemente, o deficiente suprimento de cálcio constituiu um forte fator de risco para osteoporose, já que esse nutriente desempenha importante e comprovado papel no metabolismo ósseo $3,7,8,18,25-27,30-39$.

O estudo de Lindsey et al. ${ }^{18}$, realizado com norteamericanas que tiveram câncer de mama, verificou também uma baixa ingestão dietética de cálcio (média de $673,8 \mathrm{mg}$ ), embora a prática comum de suplementação desse mineral no país tenha elevado esta média para $1353,1 \mathrm{mg} / \mathrm{dia}$. No estudo desenvolvido por Guthrie et al. ${ }^{41}$, em que foi avaliada a ingestão diária de cálcio entre mulheres australianas saudáveis, também foi encontrado que mais da metade $(52 \%)$ das mulheres ingeriam menos que $800 \mathrm{mg} /$ dia do mineral; o estudo não verificou quantas não atendiam à DRI para cálcio.

Vale ainda destacar que, analisando a Tabela 3, verifica-se que $72,9 \%$ do grupo entrevistado ingere cálcio em percentual inferior a $70 \%$ do recomendado, evidenciando que, mesmo considerando a literatura mais antiga $^{52}$, que referia ser seguro para a saúde uma ingestão diária de ao menos $70 \%$ das necessidades, as mulheres estudadas encontram-se vulneráveis à carência de cálcio. Ressalte-se também que, considerando o tipo de clientela aqui estudado, fundamental seria assegurar uma cobertura adequada de cálcio (100\%), contribuindo para a redução desse fator contribuidor para a osteoporose.

As fontes de cálcio citadas foram as mais comuns, leite principalmente $(83,1 \%$ das mulheres), com quantidades ingeridas abaixo do recomendado, como evidenciado pela baixa adequação. A quase ausência de citação de consumo de outras fontes do nutriente pode estar relacionada às condiçōes financeiras do grupo, uma vez que se trata de clientela de serviço público, habitualmente com baixos rendimentos, já que iogurtes e queijo, por exemplo, são mais onerosos. Tal monotonia na ingestão desse grupo alimentar dificulta uma boa cobertura nutricional de cálcio, pois a paciente pode não tolerar ficar repetindo diariamente 3 porções de leite, considerando que limitaçôes financeiras podem também limitar a adoção de estratégias para variar a dieta, como a adição de outros alimentos ao leite, como frutas, chocolate, entre outros.

\section{CONCLUSÃO}

O estudo evidenciou que a idade do grupo e o consumo alimentar insuficiente de cálcio constituem fatores de risco para o desenvolvimento da osteoporose, adicionais aos fatores de risco já existentes e associados aos procedimentos terapêuticos e preventivos adotados junto a sobreviventes de câncer de mama.

Os achados podem se traduzir em contribuição relevante ao atendimento desse tipo de paciente, respaldando a adoção de um protocolo de atendimento que inclua o desenvolvimento de ações educativas, com o levantamento da disponibilidade financeira para a aquisição de leite e derivados e com o incentivo para o aumento quantitativo do consumo deste grupo alimentar, sugerindo estratégias para facilitar o acesso e a aceitação do mesmo. Assim, pelo menos a ingestão dietética deficiente de cálcio como fator de risco para o desenvolvimento da osteoporose poderá ser corrigida ou, ao menos, minimizada.

\section{REFERÊNCIAS}

1. Korpelainen R, Korpelainen J, Heikkinen J, Väänänen K, Keinänen-Kiukaanniemi S. Lifestyle factors are associated with osteoporosis in lean women but not in normal and overweight women: a population-based cohort study of 1222 women. Osteoporos Int. 2003;14(1):34-43.

2. World Health Organization (WHO). Assessment of fracture risk and its application to screening for postmenopause osteoporosis. Geneva: 1994 (WHO Technical Report Series 843).

3. Pinto-Neto AM, Marinho RM, Costa-Paiva LHS, Pereira FAS, Urbanetz AA, Ferrari AEM. Osteoporose em mulheres na pós-menopausa. In: Associação Médica Brasileira / Conselho Federal de Medicina. Projeto Diretrizes. 2002;299-314.

4. Elich H. Nutritional strategies efficacious in the prevention and treatment of osteoporosis. In: American Academy of Family Physicians. Nutritional Screening Initiative. The role of nutrition in chronic disease care. Washington: American Academy of Family Physicians; 1997. 
5. Liddel DB. Cuidados aos pacientes com distúrbios musculoesqueléticos. In: Smeltzer SC, Bare BG. Tratado de enfermagem médico-cirúrgica. 8a ed. Rio de Janeiro: Guanabara Koogan; 1999:1618-621.

6. Turner RT, Sibonga JD. Effects of alcohol use and estrogen on bone. Alcohol Res Health. 2001;25(4):276-81.

7. Brown JP, Josse RG. 2002 Clinical practice guidelines for the diagnosis and management of osteoporosis in Canada. CMAJ. 2002;167(suppl) 10:1-34.

8. Follin SL, Hansen LB. Current approaches to the prevention and treatment of postmenopausal osteoporosis. Am J Health Pharm. 2003;60(9):883-901.

9. Brooks P M, Hooper M J, Smallwood R A. Bone and joint disorders: prevention and control. Med J Aust. 2004;180(suppl 5):S4-S5.

10. O'Neiil S, MacLennan A, Bass S, Diamond T, Ebeling P, Findlay D, et al. Clinical practice: guidelines for the management of postmenopausal osteoporosis for GPs. Aust Fam Physician. 2004;11:910-17.

11. Moore HC. Managing menopause after breast cancer: balancing risks and benefits. Cleve Clin J Med. 2001;68(3):243-48.

12. Arie MHA, Fonseca AMF, Arie WMY, Halbe HW, Bagnoli VR, Pinotti JA. Climatério Precoce. Rev Ginecol Obstet 2002;13(2):98-107.

13. Mortimer JE. Hormone replacement therapy and beyond. The clinical challenge of menopausal symptoms in breast cancer survivors. Geriatrics. 2002;57:25-31.

14. Welnicka-Jaskiewicz M, Jassem J. The risks and benefits of hormonal replacement therapy in healthy women and in breast cancer survivors. Cancer Treat Rev. 2003;29(5):35561.

15. Bruning PF, Pit MJ, De Jong-Bakker M, Van Den Ende A, Hart A, Van Enk A. Bone mineral density after adjuvant chemotherapy for premenopausal breast cancer. Br J Cancer. 1990;61(2):308-10.

16. Ratcliffe MA, Lanham SA, Reid DM, Dawson AA. Bone mineral density (BMD) in patients with lymphoma: the effects of chemotherapy, intermittent corticosteroids and premature menopause. Hematol Oncol. 1992;10(34):181-87.

17. Milewicz A, Szymanski W, Ruzyllo W, Zgliczynski S, Reinfus M, Zielinski J, et al. Polish consensus: procedure in case of estrogen deficiency in women after breast cancer therapy. Gynecol Endocrinol. 2002;16(5):385-89.

18. Lindsey AM, Gross G, Twiss J, Waltmwn N, Ott C, Moore TE. Postmenopausal survivors of breast cancer at risk for osteoporosis: nutritional intake and body size. Cancer Nurs. 2002;25(1):50-56.

19. Gross GJ, Ott CD, Lindsey AM, Twiss JJ, Waltman N. Postmenopausal breast cancer survivors at risk for osteoporosis: physical activity, vigor and vitality. Oncol Nurs Forum. 2002;29(9):1295-300.
20. Natrajan PK, Gambrell RD. Estrogen replacement therapy in patients with early breast cancer. Am J Obstet Gynecol. 2002;187(2):289-95.

21. Fabian CJ, Kimler BF. Chemoprevention of breast cancer: implications for postmenopausal women. Drugs Aging. 2002;19(1):43-78.

22. Baber RJ, O'Hara JL, Boyle FM. Hormone replacement therapy: to use or not to use? Med J Aust. 2003;178(12):630-33.

23. Nikander E, Metsä-Heikkilä M, Ylikorkala O, Tiitinen A. Effects of phytoestrogens on bone turnover in postmenopausal women with a history of breast cancer. J Clin Endocrinol Metab. 2004;89(3):1207-212.

24. Swenson KK, Henly SJ, Shapiro AC, Achroeder LM. Interventions to prevent loss of bone mineral density in women receiving chemotherapy for breast cancer. Clin J Oncol Nurs. 2005;9(2):177-84.

25. Susan A. Nutritional factors influencing the development and maintenance of bone health throughout the life cycle. 10p. [monograph on the internet]. World Congress on Osteoporosis, 2000. [cited $2000 \mathrm{Jul}$ 02]. Available from: <http://endocrine.medscape.com/Medscape/CNO/2000/ WCO/Story.cfm?story_fd=1402>

26. Lau EM, Woo J. Nutrition and osteoporosis. Curr Opin Rheumatol. 1998;10(4):368-72.

27. Szejnfeld VL, Castro CHM. Osteoporose. In: Waitzberg DL. Nutrição oral, enteral e parenteral na prática clínica. 3a ed. São Paulo: Atheneu; 2000:1271-280.

28. Sellmeyer DE, Stone KL, Sebastian A, Cummings SR. A high ratio of dietary animal to vegetable protein increases the rate of bone loss and the risk of fracture in postmenopausal women. Am J Clin Nutr. 2001;73:118-22.

29. Notelovitz M. Overview of bone mineral density in postmenopausal women. J Reprod Med. 2002;47(suppl.1):71-81.

30. Ilich JZ, Brownbill RA, Tamborini L. Bone and nutrition in elderly women: protein, energy and calcium as main determinants of bone mineral density. Eur J Clin Nutr. 2003;57(4):554-65.

31. Mahan LK, Escott-Stump S. Nutrição na saúde óssea. In: Krause: alimentos, nutrição e dietoterapia. 9a ed. São Paulo: Roca; 1998:583-95.

32. Levinson W, Altkorn D. Prevenção primária da osteoporose pós-menopausa. JAMA Brasil. 1999;3(10):2607-609.

33. Ullom-Minnich P. Prevention of osteoporosis and fractures. Am Fam Physician. 1999;60(1):194-202.

34. South-Paul JE. Osteoporosis: part I. Evaluation and assessment. Am Fam Physician. 2001;63(5):897-904.

35. Heaney RP. Constructive interactions among nutrients and bone-active pharmacologic agents with principal emphasis on calcium, phosphorus, vitamin D and protein. J Am Coll Nutr. 2001;20(suppl. 5):403-409; 417-20.

36. NAMS. Management of postmenopausal osteoporosis: 
position statement of the North American Menopause Society. Menopause. 2002;9(2):84-101.

37. Watts NB. Therapies to improve bone mineral density and reduce the risk of fracture: clinical trial results. J Reprod Med. 2002;47(suppl.1):82-92.

38. Malabanan AO, Holick MF. Vitamin D and bone health in postmenopausal women. J Womens Health (Larchmt). 2003;12(2):151-56.

39. Advani S, Wimalawansa SJ. Bones and nutrition: common sense supplementation for osteoporosis. Curr Womens Health Rep. 2003;3(3):187-92.

40. World Health Organization/ Food and Agriculture Organization (WHO/FAO). Expert Consultation on diet, nutrition and the prevention of chronic diseases. Geneva: 2003 (WHO Technical Report Series 916).

41. Guthrie JR, Ebeling PR, Dennerstein L, Wark JD. Risk factors for osteoporosis: prevalence, change and association with bone density. Med Gen Med. 2000;2(4). [formerly published in Medscape Womens Health. 2000;5(5).]. Available from: $<$ http://www.medscape.com/viewarticle/ 408930>

42. Yahata Y, Aoyagi K, Okano K, Yoshimi I, Kusano I, Kobayashi M, et al. Metacarpal bone mineral density, body mass index and lifestyle among postmenopausal Japanese women: relationship of body mass index, physical activity, calcium intake, alcohol and smoking to bone mineral density: the Hizen-Oshima study. Tohoku J Exp Med. 2002;196(3):123-29.

43. Roy DK, O'Neill TW, Finn JD, Lunt M, Silman AJ, Feisenberg D, et al. Determinants of incident vertebral fracture in men and women: results from the European Prospective Osteoporosis Study (EPOS). Osteoporos Int.
2003;14(1):19-26.

44. Feskanich D, Willett WC, Colditz GA. Calcium, vitamin $\mathrm{D}$, milk consumption and hip fractures: a prospective study among postmenopausal women. Am J Clin Nutr. 2003;77(2):504-11.

45. Lunt M, Masaryk P, Scheidt-Nave C, Nijs J, Poor G, Pols $\mathrm{HA}$, et al. The effects of lifestyle, dietary dairy intake and diabetes on bone density and vertebral deformity prevalence: the EVOS study. Osteoporos Int. 2001;12(8):688-98.

46. Michäelson K, Bergström R, Holmberg L, Mallmin H, Wolk A, Ljunghall S. A high dietary calcium is needed for a positive effect on bone density in Swedish postmenopausal women. Osteoporos Int. 1997;7:155-61.

47. Pinheiro ABV, Lacerda EMA, Benzecry EH, Gomes MCS, Costa VM. Tabela para avaliação de consumo alimentar em medidas caseiras. 4a ed. São Paulo: Atheneu; 2000.

48. Institute of Medicine, Food and Nutrition Board, National Academy of Sciences (IOM/FNB/NAS). Dietary reference intakes. Washington, DC: National Academy Press; 1998.

49. Phillippi ST. Tabela de composição de alimentos: suporte para decisão nutricional. 2a ed. São Paulo: Coronário; 2002.

50. Kanis JA, Johnell O, Oden A, Dawson A, De Laet C, Jonsson B. Ten year probabilities of osteoporotic fractures according to BMD and diagnostic thresholds. Osteoporos Int. 2001;12:989-95.

51. Tavani A, Pelucchi C, Parpinel M, Negri E, Franceschi S, Levi $\mathrm{F}$, et al. $\mathrm{n} 3$ poliunsaturated fatty acid intake and cancer risk in Italy and Switzerland. Int J Cancer. 2003;105:113-16.

52. Thomas PR. Guidelines for dietary planning. In: Mahan LK, Escott-Stump S. Krause's food, nutrition and diet therapy. 9th ed. Philadelphia: W.B. Saunders; 1996:331-59.

\section{Abstract}

Women who survive breast cancer show an increased risk of osteoporosis due to the effect of cancer chemotherapy and the non-utilization of hormone replacement. The current study aims to investigate whether age and dietary calcium intake are additional risk factors for the development of osteoporosis in a group of female breast cancer survivors. A total of 59 women were studied in public mastology services in Fortaleza, Ceará State, Brazil. Age 45 years or older and calcium intake less than $100 \%$ of recommended daily intake (RDI) were considered potential risk factors. According to the results, age (mean 49.14 years) and low calcium intake (detected in $86.44 \%$ of the group) appeared as contributing factors to the development of osteoporosis. In conclusion, breast cancer survivors need educational support to increase their intake of dietary calcium as a strategy to reduce osteoporosis risk factors in this group.

Key words: Osteoporosis; Breast cancer; Calcium intake 